\title{
The Design and Application of Three Component Microseismic Crack Monitor
}

\author{
Hanchuan Dong ${ }^{1, a}$, Lili Pang ${ }^{1, b}, Y u e ~ W u^{1, c}$ and Kai Yang ${ }^{1, d}$ \\ ${ }^{1}$ Center for Hydrogeology and Environmental Geology Survey, \\ CGS,CHINA \\ a359195391@qq.com,,583289675@qq.com, ${ }^{5} 553524233$ @qq.com,,1493647831 @qq.com
}

\section{Keywords: Three component; Microseismic monitoring; STM32F4; CS536; Water fracturing}

\begin{abstract}
Taking STM32F4 and CS536 chips as the core components, a three component microseismic monitoring instrument is designed, which is mainly used to capture microseismic events in shale gas exploitation. The three component geophone, which can record both longitudinal and transverse waves and converted waves, is used to sense the acceleration of particle vibration. Describes the working principle of the monitor and the hardware and software design ideas, and Jiao Shi in Fuling national demonstration area of shale gas was carried out in field experiment. Through the experimental analysis, the three component microseismic monitoring instrument can truly reflect the location of the hypocenter and the degree of the fracture, which can be used as a technical means to understand the effect of the shale gas water fracturing on the deep geological structure.
\end{abstract}

\section{Introduction}

In recent years, the development of unconventional resources has set off an upsurge in the world. Microseismic monitoring technology has become an important technology in the field of fracturing and reconstruction of low permeability oil and gas reservoirs. This technology can optimize injection and production procedures, adjust hydraulic fracturing parameters in time, correct fracturing operation procedures in time, and optimize production increase measures in the process of oil and gas reservoir mining. In order to reduce production costs and increase production of oil and gas reservoirs, the cycle and cost of oil and gas reservoir monitoring can be greatly shortened and reduced. Therefore, the microseismic monitoring technology has been widely used in the field of hydraulic fracturing fracture monitoring, oil and gas reservoir dynamic monitoring, water injection monitoring and so on, and has obtained the high recognition of the oil and gas field.

The research on microseismic monitoring technology in China started relatively late, influenced by the short time of introduction of technology and the lack of technical mastery, and the foreign technology and service were used in the field of microseismic monitoring in the field of oil and gas exploitation in China (such as Schlumberger).The domestic microseismic monitoring equipment was still in the blank. and there is no independent intellectual property rights of micro seismic monitoring equipment.

\section{General Situation and Principle}

General Situation. In the process of oil and gas exploitation, hydraulic fracturing and other engineering activities are easy to cause cracks in formation rock, and microseismic monitor is mainly used to capture the geometry and development degree of the fracture. There are two main forms of formation fracture: tensile fracture and shear fracture. Three component monitoring technology is used to monitor shear fracture.In the process of shear fracture, both longitudinal and transverse waves are produced. In each microseismic event, the longitudinal wave is in the front, the transverse wave is behind, and there are three components in the direction of propagation: two horizontal components $\mathrm{XY}$ and a vertical component $\mathrm{Z}$. The microseismic monitor realizes the location of microseismic events by collecting and analyzing three components of acceleration data. Under the support of the geological survey project of the China " The survey of geothermal 
resources in Xining and Guinan area, Qinghai ", the equipment has been carried out in Qinghai region.

Principle. Microseismic monitoring technology is a new geophysical technology which has been widely used in recent years. It is based on acoustic emission and seismology, which is mainly used to capture small earthquake events in the process of oil and gas field mining. The principle is to observe and analyze micro seismic events such as rock cracking in the deep underground of oil and gas mining activities (such as hydraulic fracturing), processing and translating the observed microseismic data and generating images, we can better understand the geometry, orientation, inclination, connectivity, density of rock cracks, etc. According to the different monitoring methods, microseismic monitoring is usually divided into two ways: observation well monitoring and surface (or near surface) monitoring. This paper adopts the latter way.

\section{Hardware Design}

The microseismic monitor uses three component geophones to perceive the three acceleration values of $\mathrm{X}, \mathrm{Y}$ and $\mathrm{Z}$, and the equipment converts the three acceleration analog signals to the identifiable signal of the CPU chip, and the data are stored in the local SD card after the memory processing and analysis. The hardware of the monitor mainly includes the embedded processor module (CPU), the power module, the filter circuit module, the program control gain amplifier module, the analog to digital converter module (AD), the GPS timing module and the SD card storage module. The structure is shown in Fig. 1. The time calibration of the system is completed by the GPS circuit. All the logic timing control in the circuit is completed by the embedded controller CPU. Each module works in collaboration with each other to capture the microseismic events.

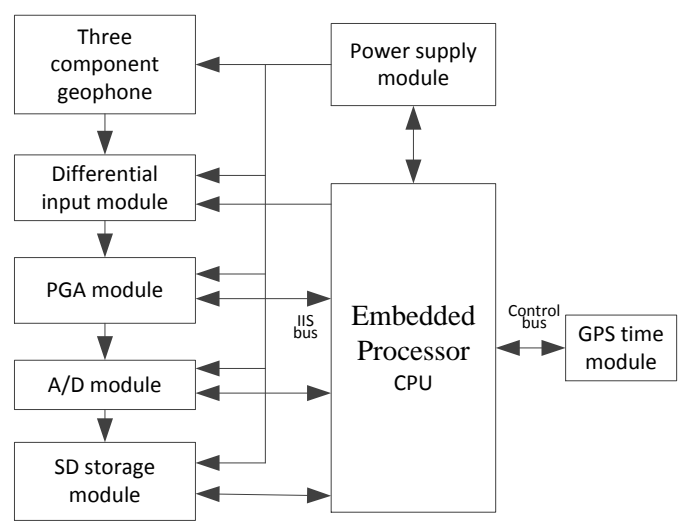

Figure 1. Finite Hardware block diagram of three component microseismic monitor

Embedded Processor CPU. The acquisition of microseismic signal is a continuous acquisition process. The amount of data is large and needs to be stored in real time. Therefore, the system needs to do as low power as possible in the selection of the system. The monitor chooses the embedded processor of STM32F4 series as the central CPU chip, the main frequency is $168 \mathrm{MHz}$, the actual ability index is $1.25 \mathrm{DMIPS} / \mathrm{MHz}$, and it has a high ability. Consumption ratio, internal integration of $256 \mathrm{~KB}$ SRAM, can meet the needs of data caching, the processor has more I/O interfaces, with IIS data ports, with CPU DMA interrupt mode, for the fast access to $\mathrm{AD}$ data, the external configuration 16M SDRAM, for caching of continuous data, the structure of which is shown in Fig. 2 . 


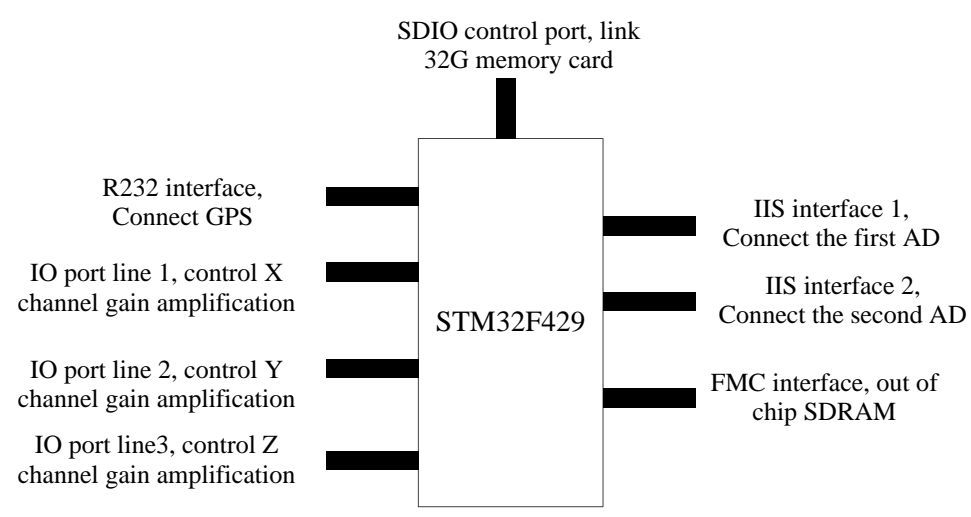

Figure 2. Finite Embedded CPU block diagram

Signal Circuit. Differential Input Circuit: The input terminal of the signal is used to suppress the common mode interference signal, and the low pass filter is used to suppress the high frequency noise. The input terminal is connected with the voltage regulator diode to prevent the entry of the static electricity from the external environment and the signal amplitude of the sensor is too large to cause the chip damage. The circuit is shown in Fig. 3.

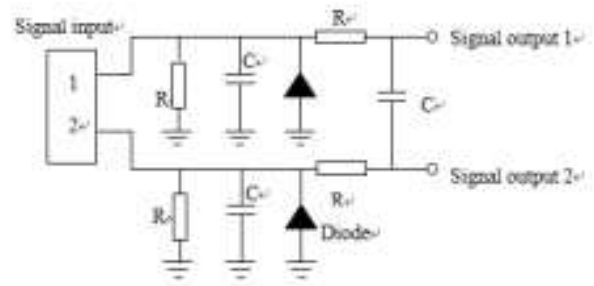

Figure 3. Finite Differential input circuit

Programmable Gain Amplification Circuit: The programmable gain amplification circuit selects PGA280AIPW as digital amplifier chip, CPU communicates with the chip through the SPI interface, the gain is adjustable, The following figures $0 \sim 10$ correspond to the magnification rate of $1 / 8$ to 128 . The output signal is further high-frequency filtering and differential filtering, reducing the signal noise. The circuit is shown in Fig. 4.

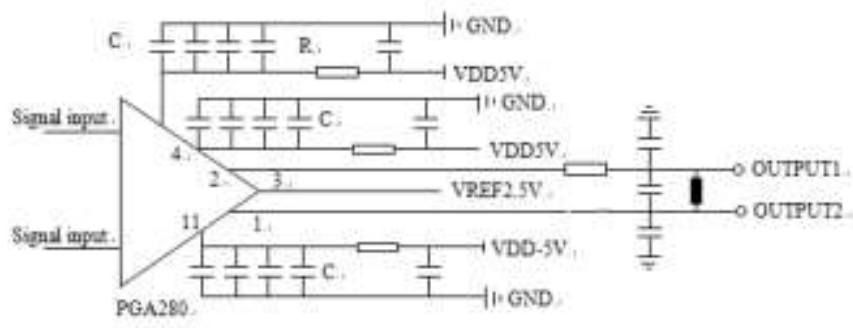

Figure 4. Finite Programmable gain amplification circuit

Analog to Digital Conversion Circuit: The analog to digital conversion circuit is the core of the microseismic signal acquisition. The circuit selects CS5361 as audio AD converter, it has $192 \mathrm{KHz}$ sampling rate, 24 bit resolution, dual channel input, and IIS data interface. After the AD is initialized, it starts to run. The data is collected in two channels at the sampling speed of $1 \mathrm{KHz}$. The data is stored in the processor ring buffer, and the trigger signal is received to the GPS school. The data of the buffer zone is moved to the external memory by the DMA manager of the processor. The speed of data processing is improved. The circuit is shown in Fig 5. 


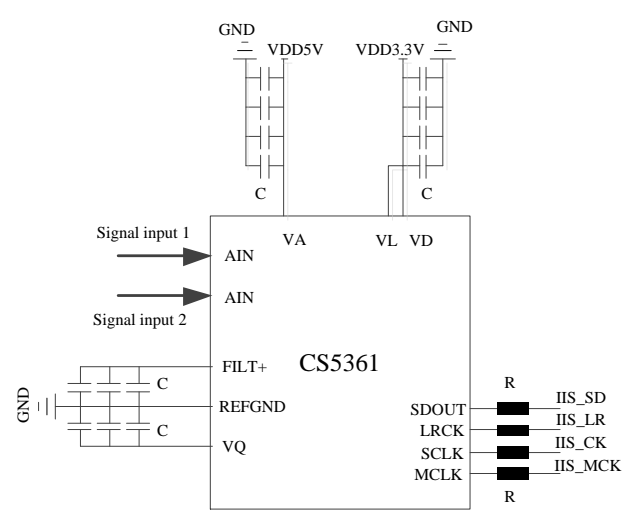

Figure 5. Finite Analog to digital conversion circuit

GPS Timing Circuit. Considering that microseismic data acquisition requires high time precision, the external oscillator of CPU chip will produce an unavoidable error after long operation. Therefore, the GPS clock module is designed as the time reference of time. Under the condition of GPS satellite signal stability, the recapture time of GPS is $1 \mathrm{~s}$, and the absolute time precision can be obtained. Up to $10^{-4}$ seconds, the second signal is used as the dividing mark of AD data length. After GPS is interrupted, the data is stored in the SD card, and the time stamp is hit in the file to ensure the consistency of the time of data acquisition.

SD Card Storage Circuit. The acquisition circuit according to the sampling speed of $1 \mathrm{KHz}, 24$ bit $\mathrm{AD}$ chip working $1 \mathrm{~s}$ will produce $4 \mathrm{~KB}$ data, a three component acquisition system is three channels, the total amount of data is $12 \mathrm{~KB}$, one hour continuous collection of $43.2 \mathrm{MB}$ data need to be stored. In this paper, the SD card with 32GB specification is selected, and the acquisition system can work continuously for about 30 days in theory. In order to prevent loss of data and facilitate subsequent data processing, the design is designed for 1 hours to generate 1 storage, and the file format is FAT32, which can be read directly under the Windows system environment.

\section{Software Design}

The hardware of the microseismic device is initialized after power, and the sequence is CPU clock, CPU interruption, SDRAM, LED indicator, SPI bus, RS232 serial, watchdog, default parameter reading, GPS, AD analog to digital converter CS5361, AD, program-controlled amplifier PGA280. After the initialization and 1 minutes of delay, GPS can already receive time information. CS5361 begins to collect data under GPS time, enables DMA channel to store a frame of data every minute, so it continues to suspend storage of data in the case of losing GPS signal. The workflow is shown in Fig.6.

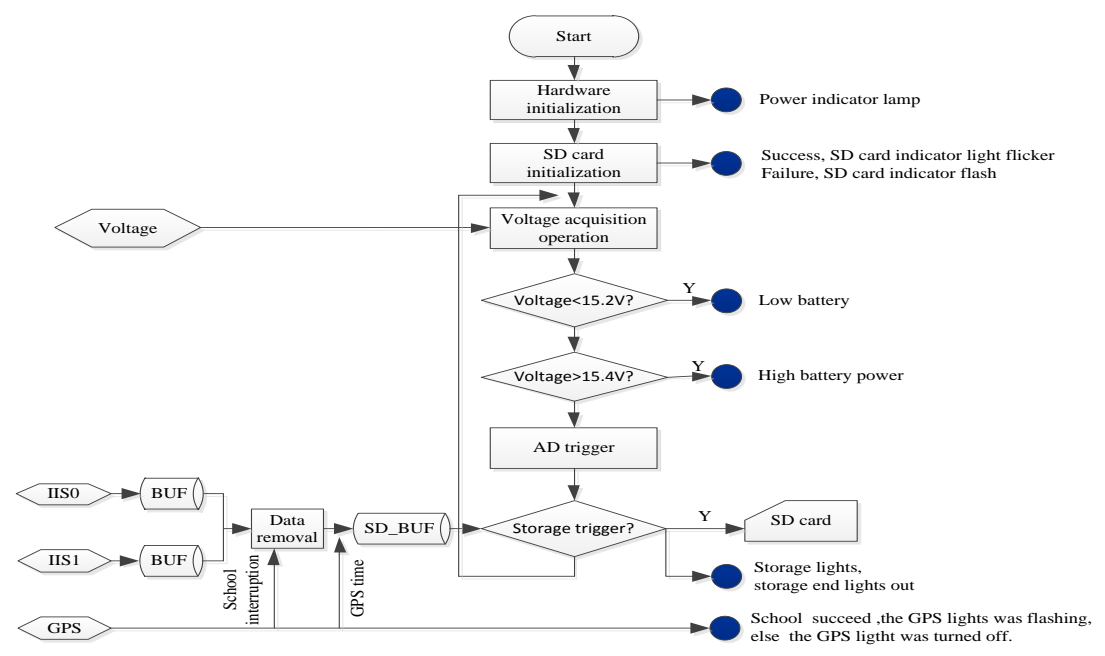

Figure 6. Finite Flow chart of system software design 


\section{Field Application Test}

The field test points for three component microseismic fracture monitoring devices are selected at geothermal monitoring wells in Qinghai. The three component monitor is equipped with a "cross shaped" array, the sensor interval is 30 meters, the length of the longitudinal and lateral monitoring lines are 570 meters, and the total amount of 39 monitoring points is set up, and the layout methods and monitoring points are shown in Fig. 7 and 8. Microseismic events are generated by artificial point sparks. The source of the source is XW5512A type spark source, with voltage 70KV. The electric spark electrode is placed in the monitoring well in the test process, and a depth is placed every 5 meters from 5 meters deep, and each depth is excited by 1 minutes at each depth, and the final depth is 50 meters for 33 microseismic monitoring, and the corresponding records are well recorded.
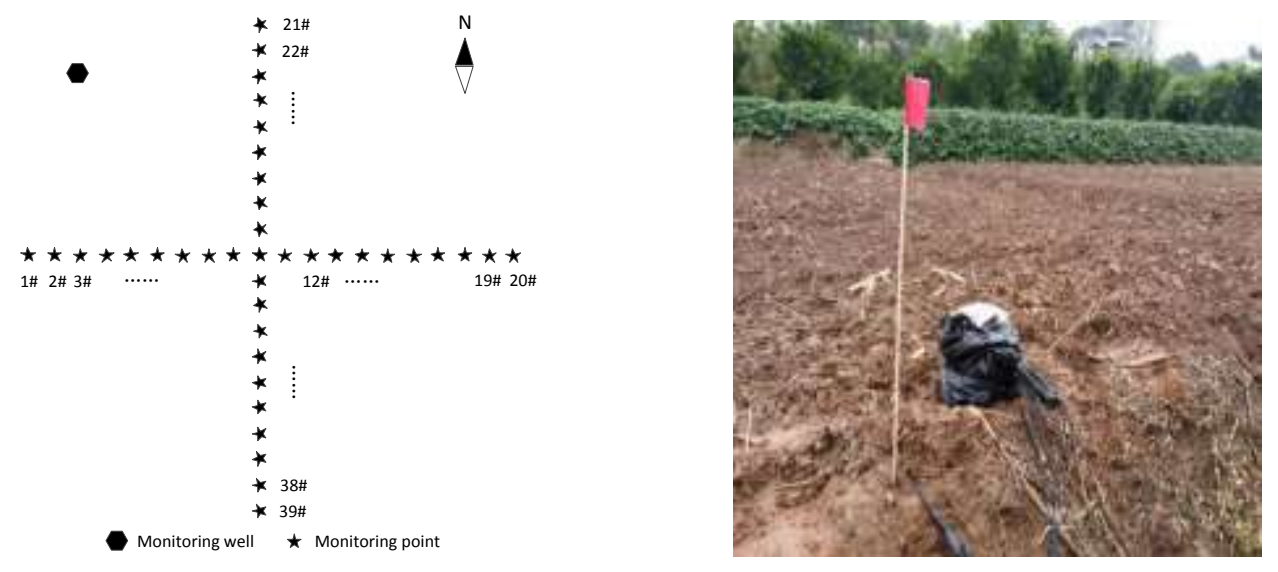

Figure 7. Finite Layout of monitoring points Figure 8. Finite Equipment on point

The 12\# microseismic monitoring points in the 45 degree direction east of the exciting point is selected for data analysis. Fig. 9 is the 21 minute data collected at 16 p.m. on November 11th, with a total of 63 records, of which 1, 2 and 3 are respectively $\mathrm{Z}, \mathrm{X}$ and $\mathrm{Y}$ components, records for 1 minutes, and the other analogies. There are more than 40 useful signals in the 21 minute record, and the rest are noise and interference signals. The filter frequency is $25-100 \mathrm{~Hz}$ bandpass filter effectively filter, filter noise and interference signal, get effective event recording curve, and analyze first effective microseismic events, as shown in Fig. 10 first to the curve, the red line position is the initial position of the longitudinal wave, of which third (Y) is better than the first, second channel to perceive the longitudinal wave ahead of time. The device collects waveform data at 16 hours and 0 minutes $120 \mathrm{~ms}$, which coincides with the actual excitation source time. The results show that the seismic source is located at the 45 degree direction of the west north of the receiving point and the vertical depth is 21.34 meters. The error between the calculated location point and the actual excitation point is vertical $1.34 \mathrm{~m}$, which is related to the initial position time of the sampled data. 


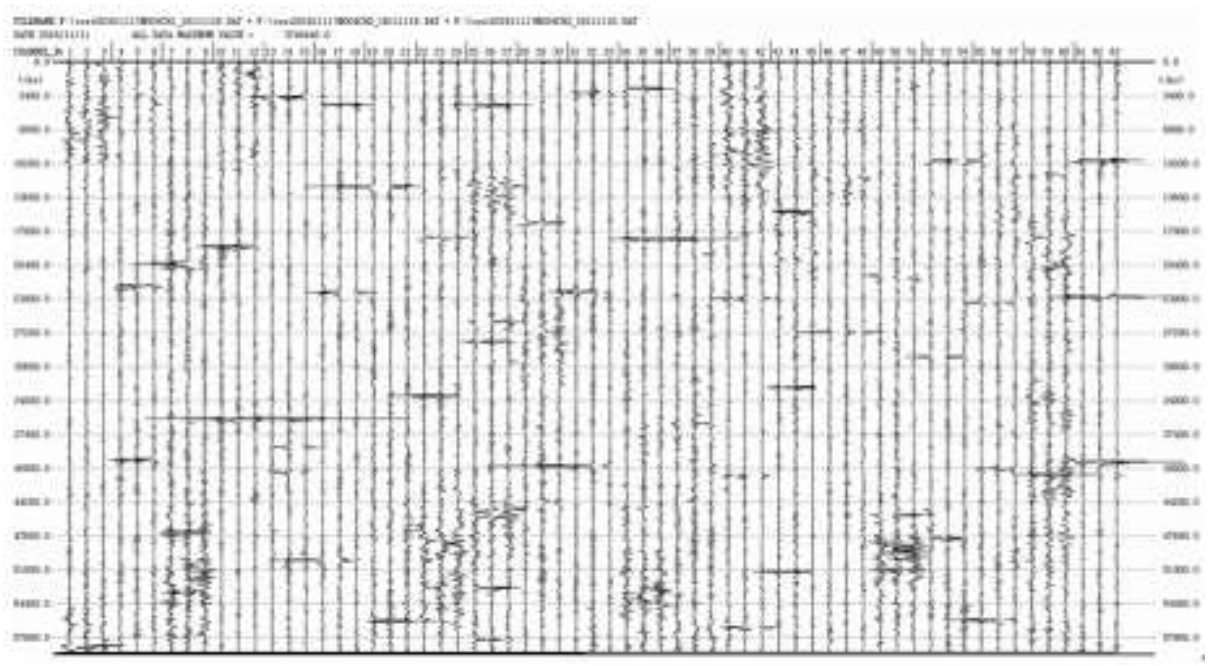

Figure 9. Finite Acquisition data of 12\# microseismic monitoring point

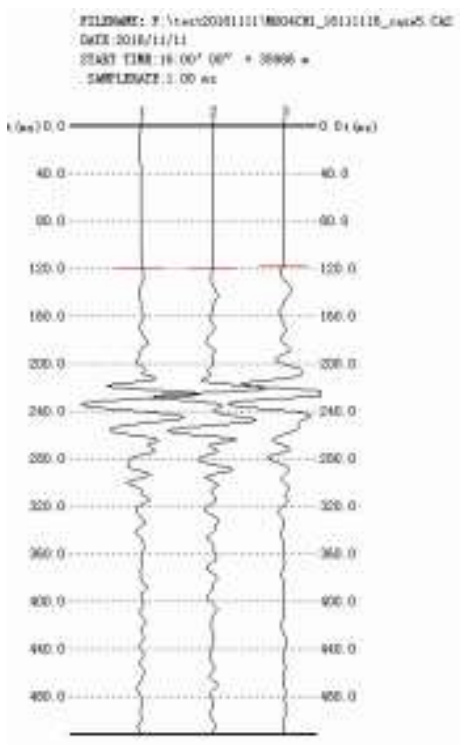

Figure 10. Finite Initial to read waveform of microseismic event

\section{Conclusion}

The three component microseismic monitor is mainly used to capture the microseismic events in the process of shale gas mining. It can reflect the location of the source, the stability and damage of the overlying strata in the target reservoir in the process of fracturing, and can be used to monitor the development and change trend of the tectonic fracture in the Ida region of the mining area. The next step is to improve the algorithm of source localization and reduce the error of equipment measurement.

\section{References}

[1] Lichun.Jia, Mian .Chen: Progress in hydraulic fracturing monitoring technology for shle gas wells abroad. Natural Gas and Petroleum 30(5), 44-47 (2012).

[2] Yonghua .Zhang, Xiang .Chen: Application of microseismic monitoring technology in horizontal well fracturing. Geophysical and Geochemical Exploration 37(6), 1080-1084(2013).

[3] Chunhua.Tang, Qingguagn.Gu: Research on microseismic monitoring technology. Engineering Science in China 14(4), 94-96 (2012). 
[4] Xue.Li,Zhihong.Zhao: Micro seismic monitoring technology and application of hydraulic fracturing. Oil Well Testing 21(3), 43-45 (2012).

[5] Hongmei.Li: Application of microseismic monitoring technology in comprehensive evaluation of fracturing effect in unconventional reservoirs. Petroleum Geology and Recovery Efficiency 22(3), 129-134 (2015).

[6] Yinlu.Duan,Qian.Li: Microseismic fracture monitoring technology for hydraulic fracturing and its application. Fault Block Oil and Gas Field 20(5),644-648 (2013).

[7] Xuli.Liu: Application of downhole microseismic monitoring technology in shale gas well factory fracturing . Petroleum Drilling Techniques 44(4), 102-107 (2016).

[8] Zhuo.Wang: A brief discussion on the monitoring technology of oil and gas development in China. Standard and quality of China's petroleum and chemical industry 31(4),205-207 (2011).

[9] Haixin.Liu: The effect of microseismic on fracturing of shale gas horizontal well. Journal of JiangHan Petroleum University of Staff and Workers 29(2), 17-32 (2016). 